\title{
15. COMMISSION POUR L'ETUDE PHYSIQUE DES COMETES
}

President: K. Wurm.

\section{Report of Meetings}

SECRETARY: Vl. Vanýsek.

Commission 15 met in three sessions, on 26, 28 and 29 August. The first session was devoted to administrative subjects including the discussion of the Draft Report, the two other meetings to a colloquium announced with the double title 'The Physics of Comets-Comets and Space Research Programmes'. The third meeting was originally not announced in the programme of Commission I 5 , it became, however, necessary since on 28 August the colloquium session had to be closed before all offered papers could have been read.

\section{Administrative Meeting, 26 August 1964}

\section{Draft Report}

The President mentioned that any errors in the Draft Report should be corrected at this meeting. He asked also to inform him about missed papers in the bibliography. The President gave a few introductory remarks to each section of the report pointing to such subjects or questions which he considered to deserve particular attention. The Draft Report was finally approved in its given form by all members. No changes were suggested beside the addition of a few references to the bibliography.

Then followed the discussion of recommendations and suggestions made by members.

(I) In a letter to the President, Dr Swings had raised the question whether there had not come the time to think of the preparation and publication of a supplement to the Atlas of Representative Cometary Spectra published nearly io years ago by Dr Swings and Dr Haser. It was mentioned in the letter that in the meantime many new spectra of comets had been obtained, and in particular such ones with high dispersion, and which prove to be of high interest for cometary research. After a short discussion of Dr Swings suggestion all members agreed to bring the following Resolution to the knowledge of the Executive Committee.

'Commisssion I 5 recommends the publication of an extension of the Atlas of Representative Cometary Spectra (published by P. Swings and L. Haser in 1955) including in particular the high dispersion spectra obtained since the publication of the Atlas.'

(2) The second suggestion which was discussed had been brought forward by Dr Elisabeth Roemer and concerns the preparation of an atlas of characteristic comet photographs.

Miss Roemer who was regrettably not able to attend the Hamburg General Assembly informed the undersigned by a letter. She suggested to prepare a representative atlas drawn from about 100 originals, and to be selected according to the following scheme:

I. Representative forms.

II. Changes with heliocentric distances.

III. Appearing of the same comet with varying exposure time, with different telescopes, different filters.

IV. Appearing of different comets at the same distance from the Sun.

V. Special effects.

Dr E. Roemer writes that the material at the Flagstaff Station, Arizona, can contribute much 
to such a plan, that, however, the scheme could not be filled exclusively by this material. A collaboration of other observatories would be necessary.

All members of the Commission I 5 who uttered their opinion to the proposal recommended the plan strongly. There was a general agreement that the following Resolution should be transferred to the Executive Committee of the IAU:

'Commission ${ }_{15}$ recommends the preparation of a photographic atlas of representative and particular comet forms and comet phenomena.

This recommendation has been adapted because of rising interest in comets as means of studying solar activity and the interplanetary medium.'

In order that the first steps towards the realization of the plan may soon be taken (after the approval of the recommendation by the Executive Committee) an organization group of some members present was set up. The same consists of Drs Donn, Dossin, Haser, Vanýsek and Wurm. This group had already a short preliminary discussion during the General Assembly, and the same decided to try next to clear the financial side of the plan, and also to explore what colleagues beside Dr Roemer may be prepared to take active part in the collection of the originals and the supervision of the copying.

(3) Dr Wurm mentioned that, by courtesy of the Astronomer Royal, Sir Richard Woolley, since some time the Greenwich photographic material of Comet Morehouse I908 III (obtained with the Greenwich 30-inch reflector) was available to him, and that this material comprises quite a number of series of six to nine photographs obtained in one and the same night and covering time intervals of 4 to 6 hours. Because such series are extremely rare and of a particular importance for a study of the dynamics of type I tails, Dr Wurm declared to intend to make some of the most interesting series more generally available by film prints. So far he had not yet been able to secure the necessary financial means for such a work. Some film copies made by a professional photographer were shown to the audience and circulated. They had a size of I5 times $20 \mathrm{~cm}$ and showed the originals about five times enlarged. The price of one single film would amount to about $\$ 0^{-75}$. Since the originals show in general a large range of blackening in order to show all details in the copies it would be necessary to make of each original at least two prints, and in many cases even three copies would be best. Dr Biermann suggested in order to avoid too long a postponement of the publication of the interesting atlas, Dr Wurm may lay open the same for subscription the costs being paid by the subscribers. This proposal was positively commended upon by several members. Dr Wurm then declared that under this condition he was prepared to start with the preparation as soon as he had found Io subscribers. He is thinking next of an atlas comprising about 60 single copies. The cost of one copy would amount to about $\$ 45$ to $\$ 5$.

(4) Suggestion no. 4 concerns an invitation to the observers of comets to collaborate within 'The International Years of Quiet Sun' programme. This invitation and suggestions concerning the way of collaboration reached the undersigned shortly before the IAU General Assembly by a letter of R. Michard, Meudon, IQSY Reporter for Solar Activity. According to the information given by R. Michard, the observations of comets were listed in the IQSY programme of a few nations, and it has been decided now by the IQSY Committee to classify comet observations with the discipline of Solar Activity. An Instruction Manual has been set up which contains recommendations to observers and describes the steps to be taken for the international collection and diffusion of the relevant data. This manual attached to $R$. Michard's letter is based on draft material prepared by the Soviet colleagues Dr Vsekhsviatsky and Dr Federov, and consultation of Dr Biermann.

As the undersigned understands it is intended to send to those who may be interested in the collaboration together with the manual five different types of forms, that is sheets of papers with specified questions. Dr Biermann showed the audience these forms in projection. 
Form no. 2 deals for instance with special questions concerning the shapes of the heads and the shapes of the tails. Form no. 5 contains questions with regard to the spectra. These forms when filled in should be sent to Dr Biermann, Munich, and also to Dr Vsekhsviatsky, Kiev. These two places are designated as 'Special World Geophysics Centres'. After processing these centres are obliged to send their results to one of the 'World Data Centres'.

Institutions and observers working in the field of comets will soon receive more detailed information.

After some discussion the following Recommendation was approved by all members present:

'Since observations of comets are likely to contribute efficiently to our understanding of the physics of interplanetary space as influenced by the radiative and corpuscular emission from the Sun, Commission I 5 greatly welcomes a systematic effort during the IQSY (International Quiet Sun Years) to insure that cometary observations are made and reported in such a way that the maximum benefit may be gained from such observations.'

There remains yet to report that there will be held at the Institut d'Astrophysique, Liège, under the chairmanship of Dr Whipple from 5 July to 7 July 1965 a symposium with the subject 'Origin and Nature of Comets'.

\section{Scientific Meetings, 28 and 29 August 1964}

COLLOQUIUM

Part 1. The Physics of Comets

\section{Contents}

(I) F.L. Whipple: On the decay of cometary nuclei.

(2) B. Donn: The chemistry of cometary atmospheres and nuclei.

(3) C. Arpigny: Recent results in cometary spectroscopy.

(4) K. Wurm: Some remarks concerning the structure of comets.

(5) E. F. Öpik: The motion of a condensation in Halley's comet.

(6) Rhea Lüst: Some results of statistical investigations of comets with plasma tails.

(7) Vl. Vanýsek: Photo-electric observations of Comet Alcock $\left(\mathrm{Ig}_{9}{ }_{3} b\right)$ during the outburst.

(8) F. Dossin: Recent observations of Comet Encke.

(9) F. D. Miller: Interpretation of surface photometry of comets.

(ro) $Y u$. B. Levin: $\mathrm{Na}$ in tails of comets.

(I ) O.V. Dubrovolskiy: On a possible mechanism of comet outbursts.

\section{Part II. Comets and Space Research Experiments}

(I) B. Donn: Possibilities of cometary research by means of space experiments.

(2) E. F. Opik: Brightness of natural and artificial comets.

(3) G. Herzberg: Lifetimes of possible parent molecules of cometary radicals.

(4) Reimar Lüst: Artificial ion cloud experiments.

(5) K. Wurm: $\mathrm{NH}_{3}$-release experiments.

The abstracts which follow have been written by the authors.

PART I. THE PHYSICS OF COMETS

I. On the decay of cometary nuclei

F. L. Whipple

Based on the concept of an icy cometary nucleus that shrinks a given fraction of its radius on each perihelion passage, a formula has been derived for the secular change in the absolute magnitude of a periodic comet. The absolute luminosity, based on the $\Delta^{-1} r^{-4}$ law, varies as 
$\mu(\mathrm{I}-\lambda t)^{2}$ where $\mu$ and $\lambda$ are constants depending upon the zero point of time, $t$, where $t>>$ period; $\mu \lambda^{2}$, however, is invariant. Vsekhsviatsky's cometary magnitudes indicate early deaths before 1970 for several comets such as Pons-Winnecke, Tuttle, Wolf I, Kopff, Brooks II, Faye and Whipple. Probably these estimates are not reliable until corrections have been applied to the magnitudes for various effects. The present results are preliminary. Eventually the rate of comet demise should provide critical information as to the manner in which icy nuclei finally become inactive or else disappear.

\section{The chemistry of cometary atmospheres and nuclei}

\section{B. Donn}

(Goddard Space Flight Center, Greenbelt, Maryland)

The major cometary problems are: (I) the nature and origin of the nucleus and (2) the mechanism of formation of observed radicals and ions. Present data indicates that photodissociation and ionization are insufficient to yield the observed short time scales of $10^{3}$ seconds. Reaction cross sections for neutral species will be several powers of 10 less than collision cross sections when the activation energy is ineluded and coma reactions will be negligible. Although ion-molecule cross sections are much larger than the neutral collision cross sections, massspectrometric data indicates that observed cometary ions will not be produced.

Whipple's icy conglomerate nucleus is the most useful basic model. Nuclear temperature would be below about $200^{\circ} \mathrm{K}$, except for very small perihelion passage. Accumulation of comets from the primordial solar nebula would have resulted in a complex composition as a result of the chemical effects of energetic radiation and the formation of metastable molecules at low temperatures. Recent research on chemical phenomena at low temperatures suggests the variety of species which can be present. Chemical energy stored in such molecules and released on warmup may account for such phenomena as cometary flares.

The normal flux of solar protons would produce a yield of $10^{-5}$ radicals per molecule. Except for periods of intense activity, no significant effects will be produced by corpuscular radiation. The formation of a crystalline lattice under interplanetary or interstellar conditions should lead to whisker-type growth of relatively pure substances rather than a complex gas-hydrate structure.

The various chemical problems described here can only be given tentative answers at present. A broad program of laboratory research is necessary to understand the structure, composition, disintegration and luminosity of comets.

Icy comet nuclei are the least modified sample of primordial material that exists in the solar system and are therefore important clues to the evolution of the solar system.

\section{Recent results in cometary spectroscopy}

\section{Observational results}

$$
\text { C. Arpigny }
$$

A general survey of the spectra of comets obtained in the last few years has been presented by Swings in a paper which will appear in the volume 7 of Advances in Space Science and Technology. At the present meeting a number of slides were shown which illustrate the progress that has been made thanks to the use of high resolution in cometary spectroscopy. Of the newer results there is to mention in particular the resolution of the violet $\mathrm{CN}$ bands and the $\mathrm{C}_{2}$ bands, further the unambiguous identification of the red doublet of $\left[\mathrm{O}_{\mathrm{I}}\right]$ of cometary origin in some comets, and the possibility to study the different intensities in different regions of the coma in the $\mathrm{CN}$ bands (so called Greenstein effect). A spectrum of Comet Ikeya (1963a) 
shows the $\mathrm{C}^{12} \mathrm{C}^{13}(\mathrm{I}, 0)$ band; the emission observed at $\lambda 4752$ is due mainly to $\mathrm{NH}_{2}$ rather than to $\mathrm{C}^{13} \mathrm{C}^{13}(\mathrm{I}, 0)$. The isotopic ratio $\mathrm{C}^{12} / \mathrm{C}^{13}$ determined by Greenstein and Stawikowski (paper in press) is essentially equal to the terrestrial ratio.

\section{Theoretical investigations}

The rotational structure of the violet $\mathrm{CN}(0,0)$ band has been studied on the basis of steadystate calculations. It is shown that much better agreement between theoretical and observed profiles is obtained when the relative population of the rotational levels are determined by solving the steady-state equations (including the effect of the Fraunhofer lines) than when these populations are assumed to be distributed according to Boltzmann's law. In reply to an objection raised in the Draft Report concerning the validity of steady-state conditions, it is pointed out that rigorous computations show that the time necessary for stationary conditions to be achieved is indeed very short compared with the characteristic time over which the excitation conditions change appreciably (for details see Ann. Astrophys. 27, 393, 1964).

Finally, it is mentioned that a detailed study of the spectrum of Comet Humason (196re) indicates that the $\mathrm{CO}^{+}$comet-tail bands are excited by the resonance-fluorescence mechanism (see Ann. Astrophys. 27, 406, 1964).

\section{Some remarks concerning the structure of comets}

\section{K. Wurm}

Next it is dealt with the deviation of the type I tails from the prolonged radius vector. From investigations of Glancy, Duncan, Kies and, in particular, Hoffmeister, we know since long, that the axes of type I tails are generally lagging behind the radius vector in the orbital motion by several degrees. The amount of this deviation of the tail from the radius vector seems to often vary considerably. Malaise and Wurm, who have recently anew investigated the effect in question, arrive at the conclusion that the position of the tail axis is determined by the forces which control the outflow of the tail ions from the vicinity of the nucleus. The second author showed some pictures of Comet Morehouse which made the situation in this respect quite clear.

Concerning the well-known turning of the tail rays (closing in) to the radius vector it is mentioned that in Comet Burnham (1959k) this process proceeded with a higher angular velocity than in other comets as for instance Comet Morehouse (I908) and Comet Brooks (I9I I). An explanation for this difference can possibly be found in a different chemical composition of the Comet Burnham tail ( $\mathrm{CH}^{+}$instead of $\mathrm{CO}^{+}$as the main constituent).

Farther on we give a brief description of the spectrographic and photographic observations of the gas and dust outburst of Comet Alcock $(1963 b)$ in May-June 1963 , as observed at the Asiago Observatory, Italy (Chincarini, Margoni, Wurm). From the expansion of a symmetrical gas halo photographed over several days an expansion velocity of the gases between $\mathrm{I} \mathrm{km}$ and $\mathrm{r} .5 \mathrm{~km} / \mathrm{sec}$ is derived. The halo consisted mainly of $\mathrm{CN}$ and $\mathrm{C}_{2}$. Later, about ro days after the appearance of the gas halo, a dust jet was becoming visible which moved with a velocity of $50 \mathrm{~m} / \mathrm{sec}$ in the direction opposite to the Sun. The jet showed as well a strong continuum as also the normal head spectrum.

Farther on we report about an investigation (carried through mainly by $U$. Eckhardt, Bergedorf, and A. Mammano, Asiago) concerning the shapes of the $\mathrm{CN}-$ and $\mathrm{C}_{2}$-heads of comets. There are strong indications that the currently used $f$-values of these molecules are too large by factors between 3 to 5 or even more. This is concluded from the observations that the $\mathrm{CN}-$ and $\mathrm{C}_{2}-$ monochromatic heads remain approximately spherical down to heliocentric distances $r=0^{\circ} 5$, whereas the influence of light pressure (assuming the presently used $f$-values) should cause a clear transition to a parabolic shape of the $\mathrm{CN}-$ and $\mathrm{C}_{2}$-heads. 


\section{The motion of a condensation in Halley's Comet}

$$
\text { E. } \text { J. Öpik }
$$

(No abstract received)

\section{Some results of statistical investigations of Comets with plasma tails \\ Rhea Lüst}

Statistical investigations of comets with plasma tails, observed between 1892 and 1957 , have been carried out by D. Antrack, L. Biermann and R. Lüst in order to study their interaction with the solar wind. The results are as follows:

(I) No dependence could be found between the number of comets with plasma tails and the general level of solar activity.

(2) Generally, plasma tails are not observed at solar distances of more than about $\mathrm{X} \cdot 5$ A.U. However, Comet Humason ( $196 \mathrm{r} e$ ) has possessed a $\mathrm{CO}^{+}$-tail already at distances of about 4-5 A.U. The comet has been in low heliocentric latitudes.

(3) No dependence could be found between the number of comets with plasma tails and their heliocentric latitude, as expressed by the latitude of their perihelia. The slight decrease towards the south pole of the ecliptic does not seem to be real. There are, however, indications for a small increase in a belt of about $\pm 15^{\circ}$ latitude around the ecliptic plane.

(4) The average of the angle between the radius vector from the Sun and the axis of the plasma tail seems to be the same for comets with direct and retrograde orbits.

These results indicate that the quiet, steady component of the solar wind is already sufficient to create and maintain a plasma tail (I). The state of the interplanetary gas does not seem to change drastically in distances beyond the Earth's orbit, at least in regions close to the ecliptic plane (2). It also seems to be the same in larger distances from the ecliptic towards the poles out to solar distances of about r.5 A.U. where most of the comets have been observed (3). There is no indication that the interplanetary gas co-rotates with the Sun in regions of more than 0.5 A.U. which is the lower limit of the solar distances of the comets investigated (4).

\section{Photo-electric observations of Comet Alcock (1963b) during the outburst}

\section{Vanýsek}

The brightness outburst of Comet Alcock $\left(\mathrm{I}_{96}{ }_{3} b\right)$ on $196_{3}$ May 29, was noted by several observers. A successful series of photo-electric observations of this phenomenon was obtained at Ondřejov Observatory (r). Scanning through the cometary head was applied in order to determine the intensity distribution in the coma and the density of the $\mathrm{CN}$ molecules. A combination of colour filters isolates the $\mathrm{CN}$ band at $3880 \AA$ and the $U$ spectral region of the $U B V$ colour system. The values of energy emitted by $\mathrm{CN}$ molecules and continuum were determined from the known spectrophotometric data of the comparison star $\beta$ Comae.

The available visual observations (2) located the maximum of outburst at May 29, but the photo-electric observations indicated the first increase in brightness-particularly the increase in band intensities-one day earlier, on May 28. During the outburst very interesting changes in intensity distribution were observed:

(I) The exponent $\kappa$ representing the surface intensity distribution by a simple formula $I=I \rho^{-\kappa}(\rho=$ distance from the nucleus) increased on May 28 from 0.75 to $\mathrm{I} \cdot \mathrm{IO}$ in an area around the nucleus of about $2 \times 10^{4} \mathrm{~km}$ diameter. At larger distances no significant change of exponent $\kappa$ was observed. On May $3^{\mathrm{I}}$ the previous mean value of $\kappa \approx 2.5$ at the periphery of the coma (about $6 \times 10^{4} \mathrm{~km}$ from the nucleus) decreased to $\approx \mathrm{I} \cdot 0$. Then the same mean value held also to the very distant area of the head of the comet. 
(2) The mean density distribution of CN molecules at a distance of $2 \times 10^{4} \mathrm{~km}$ increased on May 28 from 5 molecules per $\mathrm{cm}^{3}$ to 17 , but at a distance of about $6 \times 10^{4} \mathrm{~km}$ no increase of molecular concentration was observed. On May $3^{1}$ molecular density at $2 \times 10^{4} \mathrm{~km}$ decreased to 9 molecules per $\mathrm{cm}^{3}$, while, at the same time, at a distance of $6 \times 10^{4} \mathrm{~km}$ an increase to 5 molecules per $\mathrm{cm}^{3}$ was observed.

The above-mentioned change in surface intensity and molecular concentration may be very important for the determination of the ejection velocity of gaseous particles. The time difference between the start of an increase in the production of parent molecules from the nucleus (May 28) and the maximum of the intensity of outburst (May 31 ) was at least 24 hours. The outflow of dissociated molecules from the coma required also more than I day, as can be concluded from the density distribution on May $3 \mathrm{r}$.

If we take into consideration the unusual activity in the cometary nucleus which lasted for about 48 hours, we obtain I km sec${ }^{-1}$ for the velocity of extension of the gas in the coma. This conclusion agrees with the results mentioned by K. Wurm (3).

\section{REFERENCES}

I. Vanýsek, V., Tremko, J. Bull. astr. Inst. Csl., 15, 233, I964.

2. Millard, J. J. et al. Sky and Telescope, 26, 33, 1963.

3. Wurm, K. Draft Report of IAU, Hamburg, 1964 .

\section{Recent observations of Comet Encke F. Dossin}

During the 1964 return of Comet Encke the author was at Mt Stromlo Observatory, Australia, to study its spectrum. However, the brightness of the comet was much lower than predicted, and, in fact, the same decreased very rapidly within a few days.

July 6: the comet was seen visually through the 9 -inch $\left(f / \mathbf{I}_{5}\right)$ refractor;

July 7: a direct photograph with a 5 -inch $(f / 5)$ camera shows a diffuse image of about $m=\mathbf{I} 2$ (instead of $m=9.5$ as predicted);

July 9: guided exposure at the 5-inch camera showed a picture with a somewhat concentrated intensity of total magnitude 13.5 ; an attempt exposure for spectrum at the 74 -inch reflector with a $/ \mathrm{I}$ camera showed nothing after 24 minutes; an exposure with the Uppsala Schmidt $(f / 3.5)$ objective-prism $\left(400 \AA / \mathrm{mm}\right.$ at $\mathrm{H} \gamma$ ) revealed $\mathrm{CN}_{3} 880$ and $\mathrm{C}_{24737}$;

July ${ }_{15}$ : comet was seen at the Newton focus of the 74-inch telescope;

July r6: attempt with an 8-inch Schmidt camera $(f / r)$ to photograph the comet was without success;

July 19: a 15 minutes exposure at the 74 -inch Newtonian focus failed to show the comet; magnitude probably near $m=20$.

After that day the Moon was too bright and no other long exposure was made.

The author has requested further observations during the dark of the Moon, in order to confirm (or infirm) the rapid decrease of brightness, which may be the disparition of Comet Encke.

\section{Interpretation of surface photometry of comets}

$$
\text { F. D. Miller }
$$

The distribution of surface brightness in the head of Comet Arend-Roland (1957 III) has been studied by Miller and Dennis, using five of a series of 17 exposures taken with the Curtis Schmidt on 103a-O emulsion without filter on the night of 1957 May 5. The primary purpose was to detect any alterations in the gross distribution of light which might have accompanied rapid changes in the type I tail and the rays. No such alterations were found. 
This is the seventh detailed study of the surface brightness of this comet known to us; heretofore no attempt has been made to compare the isophote configurations with those predicted by head models.

Steady-state head models fall into two classes; those for neutral, decaying molecules experiencing negligible repulsive force; and those for dust particles which are repelled from the Sun in which, following a suggestion by Wallace and Miller, a dispersion in the velocity of ejection from the nucleus is incorporated. In both classes of models, a characteristic parameter has the dimensions of length; in the first class, the parameter is the mean distance travelled by a molecule prior to decay; in the second class, it is the most probable distance travelled by a dust particle ejected toward the Sun, before it is turned back by radiation pressure. It is noted that a number of published values of these parameters are of the order of $10^{4}$ to $10^{5} \mathrm{~km}$, although the physical significance of the two parameters is quite different.

Neither model alone is suitable for comparison with our integrated-light isophotes of Comet 1957 III, but a judicious mixture of dust and gas yields isophotes which are a satisfactory first approximation to the observed forms, to a distance of $180000 \mathrm{~km}$ toward the Sun, and $570000 \mathrm{~km}$ into the tail.

Although photometry with suitable narrow-band filters is preferable to integrated-light observations, this preliminary analysis suggests that composite dust-gas models can usefully be compared with the numerous published observations of surface brightness made in integrated light.

\section{I0. Na in tails of comets}

Yu. B. Levin

This communication appeared in the meantime in Icarus, 3, 497, 1964.

\section{1. On a possible mechanism of comet outbursts}

\section{O. V. Dobrovolskiy}

This communication, which appeared in the Bulletin of the Commission on Comets and Meteors of the Astronomical Council of the U.S.S.R. Academy of Sciences, no. 9, 3, I964, deals with enhancement of sublimation due to corpuscular bombardment (discussion of theoretical and experimental evidence with application to comets).

PART II. COMETS AND SPACE RESEARCH EXPERIMENTS

I. Possibilities of cometary research by means of space experiments

\section{B. Donn}

(Goddard Space Flight Center, Greenbelt, Maryland)

Serious gaps exist in our knowledge of comets which cannot be remedied by ground based observations. Laboratory experiments will provide certain data but some essential information will only be obtained by means of the techniques of space research.

Balloon observations with filters can search for intrinsically faint comets near the Sun or carry out standard observations as comets approach the Sun. Infra-red spectral scans using interference spectrometers and electronic Fourier transform computers are very suitable for comets because of the wide angle that can be used. The recent tentative identification of absorption bands of carbon polymers $\mathrm{C}_{4}$ through $\mathrm{C}_{12}$ in the near infra-red makes such scans extremely exciting although the fluorescent excitation mechanism becomes weak in this region.

The availability of recoverable stabilized rockets with pointing controls will permit ultraviolet photographic and spectroscopic observations of bright comets to be made. A search for faint comets in the vicinity of the Sun can be combined with observations of bright comets. $\mathrm{H}$ 
A proposed artificial comet experiment runs into the problem of the rapid mechanism of formation of the observed radicals and ions. For an assumed instantaneous production, comparison with the luminosity of the coma of equivalent linear size for actual comets indicates about tenth magnitude for a $0.5^{\circ}$ radius artificial coma from a $3 \mathrm{~m}$ diameter nucleus in a 24 hour orbit at a height of $36600 \mathrm{~km}$. With refined ground observing techniques and in situ detectors the experiment could be feasible.

The most significant space experiment and one which appears to be technically possible in the next decade is a comet probe. Present flight sensors indicate that densities of observed neutral and ionic particles in the coma would be possible at distances of about $10^{5} \mathrm{~km}$. Mass spectrometric measurements will be possible between $10^{4}$ to $10^{5} \mathrm{~km}$. Measurements of magnetic fields to $10^{-6}$ gauss and solar proton fluxes of $10^{6} \mathrm{~cm}^{-2} \mathrm{sec}^{-1}$ will permit determining the nature of the interplanetary plasma interacting with the coma.

The nucleus presents a major problem and its study should form a significant portion of the probe measurements. Photometric observations by a simple imaging device will yield sufficient resolution to determine the dimension and overall structure of the nucleus. The determination of mass and density may be possible with sensitive accelerometers.

\section{Brightness of natural and artificial comets}

$$
\text { E. F. Öpik }
$$

(No abstract received)

\section{Lifetimes of possible parent molecules of cometary radicals}

\section{G. Herzberg}

Recent observations of sudden brightness changes in comets suggest that the lifetimes of the parent molecules of $\mathrm{C}_{2}$ and $\mathrm{CN}$ in the solar radiation field are only of the order of $\mathrm{I}$ to 3 hours. The question is whether there are parent molecules that fulfil this condition. Potter and Del Duca, in a recent paper, have investigated the ultra-violet absorption spectra of a number of possible parent molecules and derived their lifetimes by combination of the absorption cross section with the solar flux of photons. They have come to the conclusion that there is no parent molecule among those studied that would yield a sufficiently short lifetime. The main point of the present paper is to point out that discrete absorptions (not considered by Potter and Del Duca for the calculation of lifetimes) may in fact be subject to slight predissociation undetectable by line broadening but still sufficient to give a high yield of dissociation. Examples of such apparently discrete spectra which under high resolution turn out to be diffuse, are the following: absorption bands of $H C N$ in the region 1900-1300 $\AA$, of ammonia in the region $2100-1400 \AA$, of ethylene in the region $175^{\circ}-155^{\circ} \AA$, and $\mathrm{H}_{2} \mathrm{O}$ near I250 $\AA$. In some of these molecules inclusion of the diffuse bands may well bring down the lifetime of the respective molecules in the solar radiation field to the required level.

The question of successive photodissociation as in $\mathrm{NH}_{3} \rightarrow \mathrm{NH}_{2} \rightarrow \mathrm{NH} \rightarrow \mathrm{N}$ and $\mathrm{CH}_{4} \rightarrow \mathrm{CH}_{3} \rightarrow \mathrm{CH}_{2} \rightarrow \mathrm{CH} \rightarrow \mathrm{C}$ is briefly discussed and it is pointed out that the lifetimes of the intermediate free radicals are probably very short. Examples are also given of cases in which in the laboratory fairly complete photodissociations of complicated molecules have been observed.

\section{Artificial ion cloud experiments \\ Reimar Lüst}

Some years ago it has been proposed by L. Biermann and R. Lüst to create an artificial ion cloud in the interplanetary space for studying the state of the interplanetary medium. New data concerning excitation and ionization by sunlight are available for the alkali-earth metals $\mathrm{Ca}$, 
$\mathrm{Sr}, \mathrm{Ba}$ and for the rare earth $\mathrm{Eu}$ which could be used for such an ion cloud. The expansion of the artificial cloud in the interplanetary space, the interaction of the ion cloud with the interplanetary medium and the minimum masses required have been estimated. The required minimum masses depend somewhat on the observational technique to be used. Estimates have been made for photographic and photo-electric methods and the results are given in the following table:

$\begin{array}{lccc} & \text { Photographic methods } & \text { Photo-electric methods } \\ \mathrm{Ca} & 33 \mathrm{~kg} & 16 \cdot 5 \mathrm{~kg} \\ \mathrm{Sr} & 3^{8} \mathrm{~kg} & 19 \mathrm{~kg} \\ \mathrm{Ba} & 4 \cdot 8 \mathrm{~kg} & 2 \cdot 4 \mathrm{~kg} \\ \mathrm{Eu} & 4 \mathrm{~kg} & 2 \mathrm{~kg}\end{array}$

Different chemical reactions have been used for the vaporization of the metals occurred. So far seven different experiments have been carried out with sounding rockets in the Sahara and in Sardinia. In these experiments metal vapour was released in the upper atmosphere.

Details can be found in paper 'Preliminary experiments for the study of the interplanetary medium by the release of metal vapour in the upper atmosphere' by H. Föppl, G. Haerendel, J. Loidl, R. Lüst, F. Melzner, B. Meyer, H. Neuss and E. Rieger, which will be published soon.

\section{5. $\mathrm{NH}_{3}$-release experiments}

\section{K. Wurm}

Together with some colleagues of the Bergedorf Observatory (H. E. Schuster, I. Hiller, J. Rahe) and further supported by Dr Mannino, Bologna, and Dr Mammano, Asiago, the author took part in the observation of the ammonia release experiments which were carried through July 6 and July 8 on the Italian island Sardinia. The project leader of the $\mathrm{NH}_{3}$-experiment was Dr Rosen, Liège. The payload was prepared by Dr Rosen and his collaborators. In the first launching a mass of $40 \mathrm{~kg}$ was released at a height of $200 \mathrm{~km}$, in the second the same mass at $180 \mathrm{~km}$.

In both experiments a rather bright cloud appeared which had instantly the shape of a symmetrical expanding ring. However, the bright phase of this expanding ring lasted only several seconds ( 3 to 4 seconds in the first launching, 6 to 7 seconds in the second one). The phenomenon was the same for other observers viewing the cloud from other directions, showing that we had to deal with an expanding shell. From the main observing station of the scientific groups (La Maddalena, north Sardinia) no trace of the cloud could be made out anymore after about 40 seconds. (After a letter of a person to the Bergedorf Observatory who viewed the cloud by chance from Tripoli, North Africa, from this place the bright expanding ring phase lasted several minutes, after which the ring had reached a diameter about 12 times that of the Moon.)

From the second launching, three well exposed spectra of the range $\lambda 4000$ to $\lambda 6800$ were obtained. The $\mathrm{NH}_{3}$-experiments were intended to investigate whether the $\mathrm{NH}_{3}$ molecules are dissociated to $\mathrm{NH}_{2}$ when the same are exposed to the unweakened $U V$ solar radiation. The $\mathrm{NH}_{2}$ molecule has a resonance spectrum in the range $\lambda_{5000}$ to $\lambda 7000$ which appears generally well developed in cometary head spectra. On the spectra obtained there appears no trace of the bands of the $\mathrm{NH}_{2}$ system, only an undisturbed scattered solar spectrum can be seen. The analysis of the physical conditions under which these spectra have been obtained (which is not yet finished) will probably allow to derive an upper limit of the product

$$
\omega\left(\mathrm{NH}_{3}-\mathrm{NH}_{2}+\mathrm{H}\right) \times p_{\mathrm{e}}
$$

wherein the factor $\omega$ designates the probability of dissociation by the $U V$ solar radiation, and $p_{\mathrm{e}}$ the probability of a fluorescence excitation of a $\mathrm{NH}_{2}$ molecule by the visible solar radiation. 